\title{
NAMES OF BACIDIA S. L. IN CURRENT USE FOR FOLIICOLOUS LICHENS - AN ANNOTATED NOMENCLATURAL STUDY
}

\author{
E. FARKAS \\ Institute of Ecology and Botany, Centre for Ecological Research \\ Hungarian Academy of Sciences, H-2163 Vácrátót, Alkotmány u. 2-4, Hungary \\ E-mail: farkas.edit@okologia.mta.hu
}

(Received 30 October, 2014; Accepted 15 December, 2014)

\begin{abstract}
The checklist contains 134 names in current use for taxa of former Bacidia s. 1. and 91 synonyms or invalid names. Place of description or new combination, basionym, synonyms, type, distribution is given for species. Type species, substrate, number of foliicolous species, systematic position, distribution is given for genera. Data are edited similarly with the online checklist on foliicolous lichens last updated by Lücking and his co-authors in 2000. The list contains the following 7 new combinations: Bacidina cinnamomea (Kremp.) Farkas, Bacidina clauzadei (Sérus. et Lambinon) Farkas, Brasilicia foliicola (Vězda) Farkas, Brasilicia ituriensis (Vězda) Farkas, Brasilicia olivaceorufa (Vain.) Farkas, Brasilicia subsimilis (Vězda) Farkas, Szczawinskia permira (Vězda) Farkas. The genus Bacidia De Not. is excluded from the checklist of foliicolous lichens, as all of its former foliicolous species are now belonging to other genera. Current names are in the following 12 genera: Bacidina, Badimia, Badimiella, Baflavia, Bapalmuia, Barubria, Brasilicia, Eugeniella, Fellhanera, Fellhaneropsis, Scoliciosporum and Szczawinskia.
\end{abstract}

Key words: Ascomycota, bacidioid taxa, lichen-forming fungi, new combinations, on leaves, synonyms

\section{INTRODUCTION}

The taxonomic and phylogenetic study of the genus Bacidia De. Not. and related taxa have been studied in various details (e.g. Ekman 1996, 2001), still several questions remained to clear for future lichenologists. In the monograph of foliicolous lichens (Santesson 1952) 33 species were mentioned to belong to the genus Bacidia. Future works by Vězda and others resulted in the description of several new Bacidia species, but also new genera and new combina- 
tions of former Bacidia species (e.g. Vězda 1974, 1975a, b, 1980, 1986). Over 200 species were mentioned as Bacidia during the last decades, which represent a large group in a wider taxonomic sense - Bacidia s. l. Most of these taxa were treated in former larger works (Lücking 2008, Lücking and Martínez Colín 2004, Lücking et al. 2000, Sérusiaux 1993) and in several scattered studies (e.g. Lücking 1998, Lücking et al. 2001). Only a few species remained which needed further nomenclatural treatment. Based on extended literature studies and microscopic anatomical and morphological investigations of herbarium material mostly of PRA-V and VBI, the necessary decisions were possible to make in case of 7 species where new combinations are published below: Bacidina cinnamomea (Kremp.) Farkas, Bacidina clauzadei (Sérus. et Lambinon) Farkas, Brasilicia foliicola (Vězda) Farkas, Brasilicia ituriensis (Vězda) Farkas, Brasilicia olivaceorufa (Vain.) Farkas, Brasilicia subsimilis (V̌̌zda) Farkas, Szczawinskia permira (Vězda) Farkas. Though the taxonomic position of a few species (e.g. Brasilicia olivaceorufa (Vain.) Farkas, Fellhanera albidocincta (Vain.) Lücking) might change in future, if further herbarium specimens will be available for study, or in other cases the transitional character sets would be evaluated in a different way, still it seems to be justifiable that these taxa cannot belong to Bacidia De Not. Therefore, as a result of this current study, no foliicolous species remained in this genus. The taxonomic position and nomenclature of taxa is changing with new results achieved by their research. New methods are developed from time to time and our knowledge must be updated accordingly. However, the synthesis of former results helps further research, especially in tropical areas where foliicolous lichens have a major role in biodiversity and bioindication studies. Foliicolous lichens of the palaeotropics are much less known, than the neotropics therefore this study might encourage the work of local researchers there too. The nomenclatural compilation below follows the structure of the online checklist of foliicolous lichens (Lücking et al. 2000). For herbarium acronyms see Index Herbariorum online (Thiers 2013). Annotations represent the place of description, combination, type, synonymy and distribution. Systematic position is given mostly according to Lumbsch and Huhndorf (2010). The checklist contains 134 names in current use for taxa of former Bacidia s. 1. and 91 synonyms or invalid names. Altogether 225 names are treated. Current names are in the following 12 genera: Bacidina, Badimia, Badimiella, Baflavia, Bapalmuia, Barubria, Brasilicia, Eugeniella, Fellhanera, Fellhaneropsis, Scoliciosporum and Szczawinskia.

The genus Bacidia De Not. is excluded from the checklist of foliicolous lichens, as all of its former foliicolous species are now belonging to other genera, just as there are no foliicolous species in Bilimbia and Woessia. 


\section{ANNOTATED LIST OF TAXA}

Bacidia De Not., Giorn. Bot. Ital. 2: 189 (1846); Ekman, Opera Bot. 127: 60 (1996). - Excluded, no foliicolous species known.

Bacidia apiahica (Müll. Arg.) Zahlbr., Catal. Lich. Univ. 4: 174 (1926); Santesson, Symb. Bot. Ups. 12(1): 442 (1952). = Bacidina apiahica (Müll. Arg.) Vězda.

Bacidia africana Vězda, Folia Geobot. Phytotax., Praha, 10: 415 (1975). ” Fellhanera africana (Vězda) Lücking.

Bacidia albidocincta (Vain.) Zahlbr., Catal. Lich. Univ. 4: 99 (1926); Santesson, Symb. Bot. Ups. 12(1): 469 (1952). झ Fellhanera albidocincta (Vain.) Lücking.

Bacidia apiahica (Müll. Arg.) Zahlbr., Catal. Lich. Univ. 4: 174 (1926); Santesson, Symb. Bot. Ups. 12(1): 442 (1952). = Bacidina apiahica (Müll. Arg.) Vězda.

Bacidia atrichoides Malme, Arkiv Bot. 27A (5): 12 (1935). - Type: Brazil, Malme 2287: 1b (S, holotype!). =Eugeniella atrichoides (Malme) Lücking, Sérus. et Kalb.

Bacidia aurantiaca Vězda, Acta Mus. Silesiae, Opava, ser. A, 23: 178 (1974).

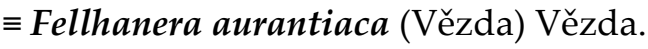

Bacidia brasiliensis (Müll. Arg.) Zahlbr., Catal. Lich. Univ. 4: 183 (1926). 三 Brasilicia brasiliensis (Müll. Arg.) Lücking, Kalb et Sérus.

Bacidia buxi Vězda et Vivant, Bull. Soc. Bot. France 119: 256 (1972). Type: France, Vivant s.n. (hb. Vězda, holotype!; Vězda, Lich. Sel. Exs. 1161, isotypes!). = Fellhaneropsis myrtillicola (Erichs.) Sérus. et Coppins, see Sérusiaux, Lichenologist 28: 204 (1996).

Bacidia carnea Vězda, Acta Mus. Silesiae, Opava, ser. A, 24: 121 (1975). ” Fellhanera carnea (Vězda) Vězda.

Bacidia cateilea (Vain.) R. Sant., Symb. Bot. Ups. 12(1): 472 (1952). =Badimia cateilea (Vain.) Lücking, Lumbsch et Elix.

Bacidia cinnamomea (Kremp.) Vain., Ann. Acad. Sci. Fenn., ser. A, 15: 65 (1921); Santesson, Symb. Bot. Ups. 12(1): 451 (1952). = Bacidina cinnamomea (Kremp.) Farkas, comb. nova.

Bacidia clauzadei Sérus. et Lambinon, Bull. Soc. Linn. Provence 45: 351 (1994). . Bacidina clauzadei (Sérus. et Lambinon) Farkas, comb. nova.

Bacidia colchica Vězda, Folia Geobot. Phytotax., Praha, 14(2): 203 (1979) $\equiv$ Fellhanera colchica (Vězda) Llop.

Bacidia consanguinea (Müll. Arg.) Zahlbr., Catal. Lich. Univ. 4: 188 (1926); Santesson, Symb. Bot. Ups. 12(1): 449 (1952). = Bapalmuia consanguinea (Müll. Arg.) Kalb et Lücking. 
Bacidia consimilis (Müll. Arg.) R. Sant., Symb. Bot. Ups. 12(1): 459 (1952). - Bas.: Calenia consimilis Müll. Arg. - Type: Costa Rica, Tonduz 5090 (G, holotype!). - Tax. syn.: non Bacidia atrichoides Malme, see Lücking 2008: 775. Distribution: Tropical America (Costa Rica, Brazil). = ?Loflammia gabrielis (Müll. Arg.) Vězda, Folia Geobot. Phytotax., Praha, 21: 216 (1986).

Bacidia corallifera Lücking, Herzogia 11: 148 (1995). =Eugeniella corallifera (Lücking) Lücking, Sérus. et Kalb.

Bacidia costaricensis Lücking, Nova Hedwigia, Beih. 104: 130 (1992); nom. inval., ICBN Art. 32, 36-37. इ Bapalmuia costaricensis Lücking et Kalb.

Bacidia depokensis Zahlbr., Ann. Crypt. Exot. 1: 181 (1928). - Type: Indonesia (Java), Schiffner 3476 (W, holotype, not seen). = Bacidia olivaceorufa Vain., see Santesson, Symb. Bot. Ups. 12(1): 452 (1952). = Brasilicia olivaceorufa (Vain.) Farkas, comb. nova.

Bacidia dimerelloides Vězda, Acta Mus. Silesiae, Opava, ser. A, 23: 179 (1974). $\equiv$ Brasilicia dimerelloides (Vězda) Farkas.

Bacidia dimidiata (Bab. ex Leight.) R. Sant., Symb. Bot. Ups. 12(1): 466 (1952). $\equiv$ Badimia dimidiata (Bab. ex Leight.) Vězda.

Bacidia dominicana (Vain.) Zahlbr., Catal. Lich. Univ. 4: 110 (1926); Santesson, Symb. Bot. Ups. 12(1): 456 (1952). - Bas.: Lecidea dominicana Vain. 三 Fellhanera fuscatula (Müll. Arg.) Vězda.

Bacidia ekmanii Vězda, Folia Geobot. Phytotax., Praha, 19: 202 (1984). $\equiv$ Fellhanera ekmanii (Vězda) Lücking.

Bacidia elegans (Vain.) Zahlbr., Catal. Lich. Univ. 4: 110 (1926); Santesson, Symb. Bot. Ups. 12(1): 463 (1952). Badimia elegans (Vain.) Vězda.

Bacidia foliicola Vězda, Acta Mus. Silesiae, Opava, ser. A, 23: 180 (1974). झ Brasilicia foliicola (Vězda) Farkas, comb. nova.

Bacidia fragilis Vězda, Folia Geobot. Phytotax., Praha, 10(4): 417 (1975) $\equiv$ Fellhanera fragilis (Vězda) Lücking et Kalb.

Bacidia fuscatula (Müll. Arg.) Zahlbr., Catal. Lich. Univ. 4: 112 (1926). ” Fellhanera fuscatula (Müll. Arg.) Vězda.

Bacidia fuscorubra Vězda, Acta Mus. Silesiae, Opava, ser. A, 24: 122 (1975).

三 Barubria fuscorubra (Vězda) Vězda.

Bacidia gabrielis (Müll. Arg.) Zahlbr., Catal. Lich. Univ. 4: 113 (1926). $\equiv$ Loflammia gabrielis (Müll. Arg.) Vězda, Folia Geobot. Phytotax., Praha, 21: 216 (1986).

Bacidia galbinea (Kremp.) Zahlbr., Catal. Lich. Univ. 4: 113 (1926); Santesson, Symb. Bot. Ups. 12(1): 462 (1952). इ Badimia galbinea (Kremp.) Vězda.

Bacidia gorgonea Vězda et Poelt in Poelt and Vězda, Herzogia 9: 241 (1992). - Type: Austria, Poelt \& Giralt s.n. (GZU, holotype!). = Fellhaneropsis myrtillicola (Erichs.) Sérus. et Coppins, see Sérusiaux, Lichenologist 28: 199 (1996). 
Bacidia ituriensis Vězda, Folia Geobot. Phytotax., Praha, 15: 84 (1980). ” Brasilicia ituriensis (Vězda) Farkas, comb. nova.

Bacidia lambinonii Sérus., Lejeunia, n.s., 90: 12 (1978). झ Fellhanera lambinonii (Sérus.) Lücking et Sérus.

Bacidia lecanorina (Zahlbr.) R. Sant., Symb. Bot. Ups. 12(1): 468 (1952). „ Badimia lecanorina (Zahlbr.) Lücking, Lumbsch et Elix, Bot. Acta 107: 400 (1994).

Bacidia leptocarpa Vain., Ann. Acad. Sci. Fenn., ser. A, 15: 65 (1921). - Type: Philippines, Robinson s.n. (TUR, lectotype). = Semigyalecta paradoxa Vain., see Santesson, Symb. Bot. Ups. 12(1): 412 (1952).

Bacidia lisowskii Vězda, Folia Geobot. Phytotax., Praha, 15: 87 (1980). ” Fellhanera lisowskii (Vězda) Vězda.

Bacidia lutea Lücking, Nova Hedwigia, Beih. 104: 130 (1992); nom. inval., ICBN Art. 32, 36-37. = Bapalmuia palmularis (Müll. Arg.) Sérus., see Lücking and Kalb, Bot. Jahrb. Syst. 122: 18 (2000).

Bacidia malayana Vain., Ann. Acad. Sci. Fenn., ser. A, 15: 73 (1921). - Type: Philippines, Robinson 17365 (TUR, holotype). = Bacidina apiahica (Müll. Arg.) Vězda, see Santesson, Symb. Bot. Ups. 12(1): 443 (1952); Vězda, Folia Geobot. Phytotax., Praha, 25: 432 (1990).

Bacidia marginalis (Vain.) R. Sant., Symb. Bot. Ups. 12(1): 445 (1952). $\equiv \mathbf{B a}$ palmuia marginalis (Vain.) Sérus.

Bacidia mastothallina Vain., Ann. Acad. Sci. Fenn., ser. A, 15: 64 (1921); Santesson, Symb. Bot, Ups. 12(1): 451 (1952). इ Bacidina mastothallina (Vain.) Vězda. $\equiv$ Fellhanera mastothallina (Vain.) Lücking et Sérus.

Bacidia michaeliana Sérus., Lejeunia, n.s., 90: 15 (1978). - Type Congo, Lambinon 71/1178 (LG, holotype!). = Fellhanera lambinonii (Sérus.) Lücking et Sérus., see Sérusiaux, Lichenologist 28: 219 (1996).

Bacidia microdiscus (Vain.) Zahlbr., Catal. Lich. Univ. 4: 126 (1926); Vězda, Acta Mus. Silesiae, Opava, ser. A, 26: 31 (1977). $\equiv$ Fellhanera microdiscus (Vain.) Vězda.

Bacidia micrommata (Kremp.) R. Sant., Symb. Bot. Ups. 12(1): 459 (1952). झ Eugeniella micrommata (Kremp.) Lücking, Sérus. et Kalb.

Bacidia myriocarpa Erichs., Mitt. Inst. All. Bot. Hamburg 10: 413 (1939). - Type: Germany, Lehr s.n. (HBG, lectotype!). = Fellhaneropsis myrtillicola (Erichs.) Sérus. et Coppins, see Sérusiaux, Lichenologist 28: 203 (1996).

Bacidia myriocarpa Vězda, Acta Mus. Silesiae, Opava, ser. A, 24: 123 (1975); nom. illeg., ICBN Art. 53.1, non Bacidia myriocarpa Erichs., see Sérusiaux, Lichenologist 28: 203 (1996). - Type: Guinea, Lisowski 1347 (hb. Vězda, holotype!; OP, UPS, isotypes!). = Fellhanera rhapidophylli (Rehm) Vězda, see Vězda, Folia Geobot. Phytotax., Praha, 15: 83 (1980). 
Bacidia “myriospora" Vězda, Folia Geobot. Phytotax., Praha, 15: 83 (1980). $=$ Orthographic error for Bacidia myriocarpa Vězda, nom. illeg.

Bacidia naevia Vain., Ann. Acad. Sci. Fenn., ser. A, 15: 66 (1921). =Fellhanera naevia (Vain.) Lücking et M. Cáceres.

Bacidia nigrescens (Müll. Arg.) Vain., Ann. Acad. Sci. Fenn., ser. A, 15: 66 (1921); Santesson, Symb. Bot. Ups. 12(1): 451 (1952). = Bapalmuia nigrescens (Müll. Arg.) Cáceres et Lücking.

Bacidia olivaceorufa Vain., Ann. Acad. Sci. Fenn., ser. A, 15: 65 (1921). Type: Philippines, Robinson s.n. (TUR, holotype, not seen). - Tax. syn.: Bacidia depokensis Zahlbr. - Distribution: Tropical Asia $\equiv$ Brasilicia olivaceorufa (Vězda) Farkas, comb. nova.

Bacidia pallidocarnea (Müll. Arg.) Zahlbr., Catal. Lich. Univ. 4: 231 (1926).

三Bacidina pallidocarnea (Müll. Arg.) Vězda.

Bacidia pallidula (Kremp.) Zahlbr., Catal. Lich. Univ. 4: 135 (1926); Santesson, Symb. Bot. Ups. 12(1): 464 (1952). इ Badimia pallidula (Kremp.) Vězda.

Bacidia palmularis (Müll. Arg.) Zahlbr., Catal. Lich. Univ. 4: 231 (1926); Santesson, Symb. Bot. Ups. 12(1): 448 (1952). = Bapalmuia palmularis (Müll. Arg.) Sérus.

Bacidia paradoxa Vězda, Acta Mus. Silesiae, Opava, ser. A, 23: 183 (1974). = Fellhanera paradoxa (Vězda) Vězda.

Bacidia pauciseptata R. Sant., Symb. Bot. Ups. 12(1): 476 (1952). झFellhanera pauciseptata (R. Sant.) Lücking.

Bacidia perminuta Vain., Ann. Acad. Sci. Fenn., ser. A, 15: 72 (1921). - Type: Philippines, Robinson s.n. (TUR, holotype, not seen). = Bacidina apiahica (Müll. Arg.) Vězda, see Santesson, Symb. Bot. Ups. 12(1): 442 (1952); Vězda, Folia Geobot. Phytotax., Praha, 25: 432 (1990).

Bacidia perminuta var. foliicola Vain., Ann. Acad. Sci. Fenn., ser. A, 15: 72 (1921). - Type: Philippines, Robinson s.n. (TUR, holotype, not seen). $=\mathbf{B a}$ cidina apiahica (Müll. Arg.) Vězda, see Santesson, Symb. Bot. Ups. 12(1): 442 (1952); Vězda, Folia Geobot. Phytotax., Praha, 25: 432 (1990).

Bacidia permira Vězda, Folia Geobot. Phytotax., Praha, 10: 419 (1975). $\equiv$ Uluguria permira (Vězda) Vězda. $\equiv$ Szczawinskia permira (Vězda) Farkas, comb. nova.

Bacidia perparva Zahlbr., Trans. New Zeal. Inst. 59: 311 (1928). = Arthonia perparva (Zahlbr.) Matzer.

Bacidia polillensis (Vain.) Zahlbr., Catal. Lich. Univ. 4: 136 (1926); Santesson, Symb. Bot. Ups. 12(1): 465 (1952). = Badimia polillensis (Vain.) Vězda.

Bacidia poliophaea Vain., Ann. Acad. Sci. Fenn., ser. A, 15: 73 (1921). - Type: Philippines, Robinson s.n. (TUR, holotype, not seen). - Tax. syn.: Bacidia mastothallina Vain., see Santesson, Symb. Bot. Ups. 12(1): 451 (1952) 三 Fellhanera mastothallina (Vain.) Lücking et Sérus. 
Bacidia psychotriae (Müll. Arg.) Zahlbr., Catalogus Lichenum Universalis 4: 137 (1926). 三 Eugeniella psychotriae (Müll. Arg.) Lücking, Sérus. et Kalb.

Bacidia rhapidophylli (Rehm) Zahlbr., Catal. Lich. Univ. 4: 138 (1926); Santesson, Symb. Bot. Ups. 12(1): 457 (1952). = Fellhanera rhapidophylli (Rehm) Vězda.

Bacidia rubicunda (Müll. Arg.) Zahlbr., Catal. Lich. Univ. 4: 239 (1926); Santesson, Symb. Bot. Ups. 12(1): 449 (1952). - Bas.: Patellaria rubicunda Müll. Arg., Lichenes epiphylli Novi: 10 (1890). इ Bapalmuia palmularis (Müll. Arg.) Sérus., see Lücking, Trop. Bryol. 13: 94 (1997); Lücking and Kalb, Bot. Jahrb. Syst. 122: 18 (2000).

Bacidia scutellifera Vězda, Folia Geobot. Phytotax., Praha, 10: 421 (1975). $\equiv$ Bacidina scutellifera (Vězda) Vězda.

Bacidia sorediantha Vězda, Folia Geobot. Phytotax., Praha, 15: 88 (1980). = Fellhanera sorediantha (Vězda) Vězda.

Bacidia spermatophora Zahlbr., Symb. Sin. 3: 118 (1930). - Type: China, Handel-Mazzetti 12276 (W, holotype, not seen). = Bacidina pallidocarnea (Müll. Arg.) Vězda, see Santesson, Symb. Bot. Ups. 12(1): 444 (1952); Vězda, Folia Geobot. Phytotax., Praha, 25: 432 (1990).

Bacidia stanhopeae (Müll. Arg.) Zahlbr., Denkschr. Math.-Nat. Kl. Akad. Wiss. Wien 81: 249 (1907); Santesson, Symb. Bot. Ups. 12(1): 474 (1952). = Fellhanera stanhopeae (Müll. Arg.) Lücking, Lumbsch et Elix.

Bacidia sublecanorina (Nyl.) Zahlbr., Catal. Lich. Univ. 4: 153 (1926); Santesson, Symb. Bot. Ups. 12(1): 475 (1952). = Fellhanera sublecanorina (Nyl.) Vězda.

Bacidia submicrommata Vězda, Acta Musei Silesiae 24: 124 (1975). इ Fellhanera submicrommata (Vězda) Lücking et Kalb.

Bacidia subsimilis Vězda, Folia Geobot. Phytotax., Praha, 10: 424 (1975). ” Brasilicia subsimilis (Vězda) Farkas, comb. nova.

Bacidia subternella (Nyl.) R. Sant., Symb. Bot. Ups. 12(1): 470 (1952). „ Fellhanera subternella (Nyl.) Vězda.

Bacidia subtilis Vězda, Preslia, Praha, 33: 367 (1961). = Fellhanera subtilis (Vězda) Diederich et Sérus.

Bacidia subundulata (Stirt.) R. Sant., Symb. Bot. Ups. 12(1): 453 (1952). ” Byssoloma subundulatum (Stirt.) Vězda.

Bacidia trichosporella Zahlbr., Denkschr. Math.-Nat. Kl. Akad. Wiss. Wien 81: 250 (1907). - Type: Western Samoa (Samoa Islands), Rechinger 1864 (W, holotype, not seen). = Bacidina pallidocarnea (Müll. Arg.) Vězda, see Santesson, Symb. Bot. Ups. 12(1): 444 (1952); Vězda, Folia Geobot. Phytotax., Praha, 25: 432 (1990). 
Bacidia tuckermanii R. Sant., Symb. Bot. Ups. 12(1): 463 (1952); nom. n. - Bas.: Lecidea palmicola Tuck., nom. illeg. = Badimia tuckermanii (R. Sant.) Lücking, Lumbsch et Elix.

Bacidia vasakii Vězda, Folia Geobot. Phytotax., Praha, 18: 64 (1983). इBacidina vasakii (Vězda) Vězda.

Bacidia vezdana Lücking, Nova Hedwigia, Beih. 104: 132 (1992); nom. inval., ICBN Art. 32, 36-37. = "Dimerella" vezdana Lücking.

Bacidia viellardii (Müll. Arg.) R. Sant., Symb. Bot. Ups. 12(1): 468 (1952). इ Badimia viellardii (Müll. Arg.) Vězda.

Bacidia wirthii Vězda, Folia Geobot. Phytotax., Praha, 15: 91 (1980). इFellhanera wirthii (Vězda) Vězda.

Bacidia ziamensis Vězda, Acta Mus. Silesiae, Opava, ser. A, 23: 184 (1974). =Bacidina ziamensis (Vězda) Vězda.

Bacidina Vězda, Folia Geobot. Phytotax., Praha, 25: 431 (1990); nom. cons. prop., see Ekman, Taxon 45: 687 (1996). - Type species (corticolous): Bacidina phacodes (Körb.) Vězda (holotype). - Tax. syn.: Lichingoldia D. Hawksw. et Poelt, nom. rej. prop.; Woessia D. Hawskw. et Poelt, nom. rej. prop. - Systematic position: Lecanorales, Ramalinaceae. - Substrata: Corticolous and foliicolous. - Number of foliicolous species: 17. - Distribution: Cosmopolitan.

Bacidina apiahica (Müll. Arg.) Vězda, Folia Geobot. Phytotax., Praha, 25: 432 (1990). - Bas.: Patellaria apiahica Müll. Arg. = Bacidia apiahica (Müll. Arg.) Zahlbr. $\equiv$ Woessia apiahica (Müll. Arg.) Sérus. - Type: Brazil, Puiggari 1025 (G, lectotype!). - Tax. syn.: Bacidia perminuta Vain.: Bacidia perminuta var. foliicola Vain.; Bacidia malayana Vain. - Exs.: Kalb, Lich. Neotrop. 477; Lücking, Lich. Fol. Exs. 56; Sipman, Lichenoth. Latinoam. 101; Vězda, Lich. Sel. Exs. 1542 (as Bacidia apiahica). - Distribution: Pantropical.

Bacidina arvidssonii (Sérus.) Lücking, Flora Neotropica 103: 608 (2008). - Bas.: Woessia arvidssonii Sérus. - Type: Ecuador, Arvidsson \& Nilson 290 (GB, holotype!). - Distribution: Tropical America.

Bacidina canariensis Lumbsch et Vězda, Lichenologist 24: 22 (1992). झ Woessia canariensis (Lumbsch et Vězda) Sérus. - Type: Spain (Canary Islands), Lumbsch \& Mietzsch 7227 (hb. Lumbsch, holotype, not seen; hb. Vězda, isotype!). - Distribution: Europe (Canary Islands, Madeira).

Bacidina cinnamomea (Kremp.) Farkas, comb. nova, Acta Bot. Hung. 57(12): 00 (2015). - Bas.: Lecidea cinnamomea Kremp., Lichenes foliicoloae quos legit O. Beccari annis 1866-1867 in insula Borne: 7 (1874); Nuovo Gior. Bot. Ital. 7: 28 (1875). = Bacidia cinnamomea (Kremp.) Vain., Ann. Acad. Sci. Fenn., ser. A, 15: 65 (1921); Santesson, Symb. Bot. Ups. 12(1): 451 (1952). - Type: Malaysia (Borneo), Beccari 226 (M, holotype!; G, isotype!). - Distribution: Tropical Asia.

Bacidina clauzadei (Sérus. et Lambinon) Farkas, comb. nova, Acta Bot. Hung. 57(1-2): 00 (2015). - Bas.: Bacidia clauzadei Sérus. et Lambinon, Bull. Soc. 
Linn. Provence 45: 351 (1994). - Type: Congo, Lambinon 78/262 (LG, holotype!). - Distribution: Tropical Africa (Congo).

Bacidina defecta Vězda, Nova Hedwigia 58: 124 (1994). $\equiv$ Woessia defecta (Vězda) Sérus. - Type: Tanzania, Pócs 6438 (hb. Vězda, holotype!). - Distribution: Pantropical.

Bacidina hypophylla Lücking et Kalb, Bot. Jahrb. Syst. 122: 17 (2000). Type: Brazil, Kalb \& Kalb 32502 (hb. Kalb, holotype!). - Distribution: Tropical America.

Bacidina mastothallina (Vain.) Vězda in Vězda et al., Ann. Naturh. Mus. Wien 99B: 738 (1997). - Bas.: Bacidia mastothallina Vain. $\equiv$ Fellhanera mastothallina (Vain.) Lücking et Sérus.

Bacidina mirabilis (Vězda) Vězda, Folia Geobot. Phytotax., Praha, 25: 432 (1990). - Bas.: Catillaria mirabilis Vězda, Folia Geobot. Phytotax., Praha, 15: 80 (1980). - Type: Congo, Lisowski s.n. (hb. Vězda, holotype!). - Exs.: Lücking, Lich. Fol. Exs. 217; Vězda, Lich. Sel. Exs. 2458. - Distribution: Tropical America and Africa.

Bacidina neotropica Lücking, Flora Neotropica 103: 608 (2008). - Type: Guyana, Lücking 96-3785 (BRG holotype; isotype, LG). - Distribution: Neotropical.

Bacidina pallidocarnea (Müll. Arg.) Vězda, Folia Geobot. Phytotax., Praha, 25: 432 (1990). - Bas.: Patellaria pallidocarnea Müll. Arg., Flora 64: 232 (1881). = Bacidia pallidocarnea (Müll. Arg.) Zahlbr. - Type: Brazil, Puiggari 492 (G, holotype!). - Tax. syn.: Patellaria translucens Müll. Arg.; Bacidia trichosporella Zahlbr.; Bacidia spermatophora Zahlbr. - Exs.: Lücking, Lich. Fol. Exs. 29. Distribution: Pantropical.

Bacidina pseudohyphophorifera (Lücking et Sérus.) Lücking, Flora Neotropica 103: 607 (2008). - Type: Lesser Antilles (St. Lucia), Sérusiaux s.n. (LG, holotype!). - Distribution: Tropical America and Asia (Hong Kong). = Woessia pseudohyphophorifera Lücking et Sérus. in Sérusiaux, Biblioth. Lichenol. 58: 422 (1995).

Bacidina scutellifera (Vězda) Vězda, Folia Geobot. Phytotax., Praha, 25: 432 (1990). - Bas.: Bacidia scutellifera Vězda. - Type: Tanzania, Pócs 6438 (hb. Vězda, holotype!; Vězda: Lich. Sel. Exs. 1310, isotypes!). - Exs.: Vězda, Lich. Sel. Exs. 1310. - Distribution: Pantropical.

Bacidina simplex Farkas et Vězda, Folia Geobot. Phytotax., Praha, 28: 321 (1993). - Type: Tanzania, Farkas 89102 (VBI, holotype!). - Distribution: Tropical Africa (Tanzania).

Bacidina simplex fo. cyanophila Lücking, Flora Neotropica 103: 603 (2008). - Type: Colombia, Sipman \& Duivenvoorden 28015 (holotype, B). Distribution: Tropical America. 
Bacidina streimannii Vězda, Nova Hedwigia 58: 125 (1994). - Type: Papua New Guinea (New Guinea), Streimann 8212017 (CANB, holotype, not seen). - Distribution: Tropical Asia (Papua New Guinea).

Bacidina vasakii (Vězda) Vězda, Folia Geobot. Phytotax., Praha, 25: 432 (1990). - Type: Georgia, Vězda s.n. (hb. Vězda, holotype!; Vězda, Lich. Sel. Exs. 1869, isotypes!). - Exs.: Vězda, Lich. Sel. Exs. 1869. - Distribution: Europe (Georgia).

Bacidina ziamensis (Vězda) Vězda, Folia Geobot. Phytotax., Praha, 25: 432 (1990). - Type: Guinea, Lisowski 1254 (hb. Vězda, holotype!; UPS, isotype!). - Distribution: Tropical Africa.

Badimia Vězda, Folia Geobot. Phytotax., Praha, 21: 206 (1986); nom. cons. prop., see Lücking and Vězda, Taxon 44: 227 (1995). - Type species: Badimia dimidiata (C. Bab. ex Leight.) Vězda (holotype). - Tax. syn.: Pseudogyalecta Vězda, nom. rej. prop. - Systematic position: Lecanorales, Pilocarpaceae, see Sérusiaux, Lichenologist 18: 6 (1986); Lücking et al., Bot. Acta 107: 399 (1994). - Substrata: Predominantly foliicolous. - Number of foliicolous species: 13. - Distribution: Pantropical.

Badimia cateilea (Vain.) Lücking, Lumbsch et Elix, Bot. Acta 107: 400

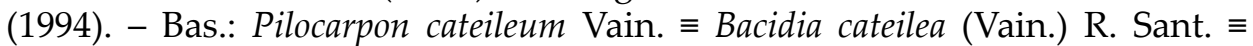
Fellhanera cateilea (Vain.) Farkas. - Type: French Polynesia (Society Islands), Setchell \& Parks 5142 (TUR, lectotype!; PC, UPS, W, isotypes!). - Distribution: Tropical Asia.

Badimia dimidiata (C. Bab. ex Leight.) Vězda, Folia Geobot. Phytotax., Praha, 21: 215 (1986). - Bas.: Lecanora dimidiata C. Bab. ex Leight. $\equiv$ Bacidia dimidiata (C. Bab. ex Leight.) R. Sant. - Type: Brazil, Spruce 113 (BM ex K, holotype!). - Tax. syn.: Patellaria subpulchra Müll. Arg.; Patellaria sorocula Müll. Arg.; Patellaria leioplacella Müll. Arg. - Exs.: Kalb, Lich. Neotrop. 252 (as Bacidia dimidiate); Lücking, Lich. Fol. Exs. 106, 130; Sipman, Lichenoth. Latinoam. 52. - Distribution: Tropical America and Africa.

Badimia elegans (Vain.) Vězda, Folia Geobot. Phytotax., Praha, 21: 215 (1986). - Bas.: Bilimbia elegans Vain. = Bacidia elegans (Vain.) Zahlbr. - Type: Philippines, Robinson s.n. (TUR-Vainio 21579, lectotype!; UPS, isolectotype!). - Distribution: Tropical Africa and Asia.

Badimia elixii Lücking, Kalb et Lumbsch, Bibliotheca Lichenologica 78: 252 (2001). - Type New Caledonia, K. Kalb \& A. Kalb s.n. (hb. Kalb, holotype; isotypes - R. Lücking, Lich. Fol. Exs. 131, as B. tuckermanii). - Distribution: Eastern paleotropical.

Badimia galbinea (Kremp.) Vězda, Folia Geobot. Phytotax., Praha, 21: 215 (1986). - Bas.: Lecidea galbinea Kremp. झ Bacidia galbinea (Kremp.) Zahlbr. - Type: Malaysia (Borneo), Beccari 220a (M, holotype!; G, isotype!). - Exs.: Lücking, Lich. Fol. Exs. 5, 57, 107. - Distribution: Tropical America and Asia. 
Badimia lecanorina (Zahlbr.) Lücking, Lumbsch et Elix, Bot. Acta 107: 400 (1994). - Bas.: Pilocarpon lecanorinum Zahlbr. = Bacidia lecanorina (Zahlbr.) R. Sant. $\equiv$ Fellhanera lecanorina (Zahlbr.) Farkas. - Type: Western Samoa (Samoa Islands), Rechinger 3390 (W, holotype!; C, S, UPS, isotypes!). - Exs.: Anonymous, Krypt. Exs. Vindob. 1529 (as Pilocarpon lecanorinum). - Distribution: Tropical Asia (Samoa Islands).

Badimia leioplacella (Müll. Arg.) Lücking, Flora Neotropica 103: 724 (2008). - Bas.: Patellaria leioplacella Müll. Arg., J. Linn. Soc. Bot. 29: 324. 1892. - Type: Brazil, Spruce 470 (holotype, BM ex K; isotypes, G, UPS). - Tax. syn: Badimia subelegans Sipman et Lücking, Trop. Bryol. 15: 54 (1998). - Distribution: Tropical America.

Badimia montoyana Lücking in Lücking and Lücking, Herzogia 11: 152 (1995). - Type: Costa Rica (Cocos Isl.), Lücking \& Lücking 92-724 (M, holotype!; CR, G, GZU, LG, ULM, UPS, Lücking, Lich. Fol. Exs. 129, isotypes!). Exs.: Lücking, Lich. Fol. Exs. 129. - Distribution: Tropical America.

Badimia pallidula (Kremp.) Vězda, Folia Geobot. Phytotax., Praha, 21: 215 (1986). - Bas.: Lecidea pallidula Kremp. झ Bacidia pallidula (Kremp.) Zahlbr. - Type: Malaysia (Borneo), Beccari 232 (M, holotype!; G, isotype!). - Tax. syn.: Bilimbia polillensis var. parcior Vain. - Exs.: Lücking, Lich. Fol. Exs. 58; Vězda, Lich. Rar. Exs. 423. - Distribution: Tropical America and Asia.

Badimia polillensis (Vain.) Vězda, Folia Geobot. Phytotax., Praha, 21: 215 (1986). - Bas.: Bilimbia polillensis Vain. $\equiv$ Bacidia polillensis (Vain.) Zahlbr. - Type: Philippines, Robinson s.n. (TUR-Vainio 21548, holotype!). - Tax. syn.: Bilimbia polillensis var. interrupta Vain.; Bilimbia polillensis var. pallescens Vain. - Distribution: Pantropical.

Badimia stanhopeae (Müll. Arg.) Vězda, Sched. Lich. Sel. Exs., Fasc. XCIII: 2, no. 2307 (1989). इ Fellhanera stanhopeae (Müll. Arg.) Lücking, Lumbsch et Elix.

Badimia subelegans Sipman et Lücking in Lücking, Trop. Bryol. 15: 54 (1998). = Badimia leioplacella (Müll. Arg.) Lücking.

Badimia tuckermanii (R. Sant.) Lücking, Lumbsch et Elix, Bot. Acta 107: 400 (1994). - Bas.: Lecidea palmicola Tuck., nom. illeg. = Bacidia tuckermanii R. Sant., nom. n. - Type: Cuba, Wright 201 (FH, holotype, not seen; G, UPS, isotypes!). - Distribution: Tropical America.

Badimia verrucosa (Vězda) Lücking et Vězda, Flora Neotropica 103: 719 (2008). - Bas.: Pseudogyalecta verrucosa Vězda, Folia Geobot. Phytotax., Praha, 10: 408 (1975). - Type: Tanzania, Pócs 6870 (hb. Vězda, holotype!). - Distribution: Tropical Africa.

Badimia viellardii (Müll. Arg.) Vězda, Folia Geobot. Phytotax., Praha, 21: 215 (1986). - Bas.: Lecania viellardii Müll. Arg. = Bacidia viellardii (Müll. Arg.) 
R. Sant. - Type: New Caledonia, Viellard 2798 (G, holotype!). - Tax. syn.: Bilimbia rizalensis Vain. - Distribution: Tropical Asia and Australia.

Badimia sect. Pseudogyalecta (Vězda) Lücking et Vězda, Flora Neotropica 103: 721 (2008). - Type species: Pseudogyalecta verrucosa Vězda $\equiv$ Badimia verrucosa (Vězda) Lücking et Vězda.

Badimiella Malcolm et Vězda, Nova Hedwigia 59: 518 (1994). - Type species: Badimiella serusiauxii Malcolm et Vězda (holotype). - Systematic position: Lecanorales, Pilocarpaceae. - Substrata: Foliicolous. - Number of foliicolous species: 1. - Distribution: Tropical Australia (New Zealand).

Badimiella serusiauxii Malcolm et Vězda, Nova Hedwigia 59: 519 (1994). - Type: New Zealand, Malcolm 1164 (CHR, holotype, not seen; hb. Vězda, isotype!). - Distribution: Tropical Australia (New Zealand).

Baflavia Lücking, Flora Neotropica 103: 718 (2008). - Type species: Baflavia flavescens (Lücking) Lücking. - Systematic position: Lecanorales, Pilocarpaceae, see Lücking, Flora Neotropica 103: 826 (2008). - Substrata: Foliicolous. - Number of foliicolous species: 1. - Distribution: Tropical America (Costa Rica).

Baflavia flavescens (Lücking) Lücking, Flora Neotropica 103: 718 (2008). - Bas.: Barubria flavescens Lücking: - Type: Costa Rica, Lücking 92-328 (CR, holotype!; VBI, Lücking, Lich. Fol. Exs. 230, isotypes!). - Exs.: Lücking, Lich. Fol. Exs. 230. - Distribution: Tropical America (Costa Rica).

Bapalmuia Sérus., Nord. J. Bot. 13: 449 (1993). - Type species: Bapalmuia palmularis (Müll. Arg.) Sérus. (holotype). - Systematic position: Lecanorales, Pilocarpaceae, see Ekman, Opera Bot. 127: 39 (1996); Kalb et al., Mycotaxon 75: 284 (2000). - Substrata: Foliicolous. - Number of foliicolous species: 14. Distribution: Pantropical.

Bapalmuia araracuarensis Sipman et Lücking, Flora Neotropica 103: 671 (2008). - Type: Colombia. Caquetá: NE of Araracuara, 250 m, Oct 1988, Sipman \& Duivenvoorden 27980 (holotype, B; isotype, hb. Lücking). - Distribution Tropical America.

Bapalmuia consanguinea (Müll. Arg.) Kalb et Lücking in Lücking and Kalb, Bot. Jahrb. Syst. 122: 20 (2000). - Bas.: Patellaria consanguinea Müll. Arg. 三 Bacidia consanguinea (Müll. Arg.) Zahlbr. - Type: Brazil, Puiggari 2288 (G, lectotype!). - Tax. syn.: Lecidea endoporphyra Vain. - Distribution: Tropical America (southern Brazil).

Bapalmuia costaricensis Lücking et Kalb in Kalb et al., Mycotaxon 75: 91 (2000). झ Bacidia costaricensis Lücking, nom. inval. - Type: Costa Rica, Lücking 91-1835 (CR, holotype!). - Distribution: Tropical America.

Bapalmuia ivoriensis R. Sant. et Lücking, Nord. J. Bot. 19: 725 (1999). Type: Ivory Coast, Santesson 10668: 7 (UPS, holotype!). - Distribution: Tropical Africa. 
Bapalmuia kakouettae Sérus., Nord. J. Bot. 13: 449 (1993). - Type: France, Sérusiaux 7703 (LG, holotype!). - Tax. syn.: Byssoloma aptrootii Sérus. - Distribution: Europe.

Bapalmuia lineata Lücking et Kalb in Kalb et al., Mycotaxon 75: 296 (2000). - Type: Costa Rica, Lücking 91-1266 (CR, holotype!). - Exs.: Lücking, Lich. Fol. Exs. 222. - Distribution: Tropical America.

Bapalmuia marginalis (Vain.) Sérus., Nord. J. Bot. 13: 451 (1993). - Bas.: Helotiopsis marginalis Vain. $\equiv$ Bacidia marginalis (Vain.) R. Sant. - Type: Philippines, Robinson s.n. (TUR-Vainio 26083, holotype!). - Distribution: Tropical Asia.

Bapalmuia napoensis Lücking, Flora Neotropica 103: 675 (2008). - Type: Ecuador. Napo: Jatun Satcha Biological Station, 450 m, May 1996, Lücking 96984 (holotype, QCNE). - Distribution: Tropical America (Ecuador).

Bapalmuia nigrescens (Müll. Arg.) Cáceres et Lücking, Nova Hedwigia 70: 218 (2000). - Bas.: Patellaria nigrescens Müll. Arg. 三 Bacidia nigrescens (Müll. Arg.) Vain. - Type: Brazil, Glaziou 18100 (G, lectotype!). - Distribution: Tropical America.

Bapalmuia pallescens Lücking, Flora Neotropica 103: 674 (2008). - Type: Brazil, Pará: Near Belém, 10 m, Oct 1980, Kalb 32717 (hb. Kalb). Caxiuanã Field Station, 25 m, May 1995, Lücking 95-192 (holotype, MG).

Bapalmuia palmularis (Müll. Arg.) Sérus., Nord. J. Bot. 13: 451 (1993). - Bas.: Patellaria palmularis Müll. Arg. = Bacidia palmularis (Müll. Arg.) Zahlbr. - Type: Brazil, Glaziou 18069 (G, holotype!). - Tax. syn.: Patellaria brasiliensis var. laevis Müll. Arg.; Patellaria rubicunda Müll. Arg. = Bapalmuia rubicunda (Müll. Arg.) Sérus.; Bacidia lutea Lücking, nom. inval. - Exs.: Lücking, Lich. Fol. Exs. 221; Vězda, Lich. Sel. Exs. 2031. - Distribution: Pantropical.

Bapalmuia rubicunda (Müll. Arg.) Sérus., Nord. J. Bot. 13: 451 (1993). - Bas.: Patellaria rubicunda Müll. Arg. = Bapalmuia palmularis (Müll. Arg.) Sérus.

Bapalmuia sorediata Kalb et Lücking in Kalb et al., Mycotaxon 75: 303 (2000). - Type: Australia, Kalb \& Kalb 26820 (CANB, holotype!; hb. Kalb, isotype!). - Distribution: Tropical Australia.

Bapalmuia variratae Sérus. in Kalb et al., Mycotaxon 75: 304 (2000). Type: Papua New Guinea (New Guinea), Sérusiaux s.n. (LG, holotype!). Distribution: Tropical Asia (Papua New Guinea).

Bapalmuia verrucosa Sérus. et Lücking in Lücking et al., Lichenologist 30: 177 (1998). - Type: Brazil, Peres 15486 (URM 22249, holotype!). - Distribution: Tropical America (northern South America).

Barubria Vězda, Folia Geobot. Phytotax., Praha, 21: 207 (1986). - Type species: Barubria fuscorubra (Vězda) Vězda (holotype). - Systematic position: Lecanorales, Ectolechiaceae, see Vězda, Folia Geobot. Phytotax., Praha, 21: 
201 (1986). - Substrata: Foliicolous. - Number of foliicolous species: 1. - Distribution: Pantropical.

Barubria flavescens Lücking, Phyton (Horn, Austria) 39: 134 (1999). - Type: Costa Rica, Lücking 92-328 (CR, holotype!; VBI, Lücking, Lich. Fol. Exs. 230, isotypes!). - Exs.: Lücking, Lich. Fol. Exs. 230. - Distribution: Tropical America (Costa Rica). = Baflavia flavescens (Lücking) Lücking.

Barubria fuscorubra (Vězda) Vězda, Folia Geobot. Phytotax., Praha, 21: 215 (1986). - Bas.: Bacidia fuscorubra Vězda. - Type: Guinea, Lisowski 1341 (hb. Vězda, holotype!; OP, UPS, isotypes!). - Exs.: Lücking, Lich. Fol. Exs. 229. Distribution. Tropical America and Africa.

Bilimbia De Not., Giorn. Bot. Ital. 2: 190 (1846). = Mycobilimbia Rehm. Excluded, no foliicolous species known. - For synonymous Bilimbia names see Lücking et al. (2000).

Brasilicia Lücking, Kalb et Sérus., Flora Neotropica 103: 667 (2008). Type species: Brasilicia brasiliensis (Müll. Arg.) Lücking, Kalb et Sérus., Flora Neotropica 103: 667 (2008). = Patellaria brasiliensis Müll. Arg., Lichenes Epiphylli Novi: 10 (1890). - Systematic position: Lecanorales, Pilocarpaceae. Substrata: foliicolous. - Number of foliicolous species: 6. - Distribution: Pantropical.

Brasilicia brasiliensis (Müll. Arg.) Lücking, Kalb et Sérus., Flora Neotropica 103: 667 (2008). - Bas.: Patellaria brasiliensis Müll. Arg., Lichenes Epiphylli Novi: 10 (1890). = Bacidia brasiliensis (Müll. Arg.) Zahlbr., Catal. Lich. Univ. 4: 183 (1926). - Type: Brazil, Puiggari 1086 (G, lectotype!). - Exs.: Kalb, Lich. Neotrop. 1, 2; Lücking, Lich. Fol. Exs. 4. - Distribution: Tropical America.

Brasilicia dimerelloides (Vězda) Farkas, Acta Bot. Hung. 57(1-2): 00 (2015). - Bas.: Bacidia dimerelloides Vězda, Acta Mus. Silesiae, Opava, ser. A, 23: 179 (1974). - Type: Guinea, Lisowski 1228 (hb. Vězda, holotype!; UPS, isotype!). - Exs.: Vězda, Lich. Sel. Exs. 2159, 2376. - Distribution: Tropical Africa.

Brasilicia foliicola (Vězda) Farkas, comb. nova, Acta Bot. Hung. 57(1-2): 00 (2015). - Bas.: Bacidia foliicola Vězda, Acta Mus. Silesiae, Opava, ser. A, 23: 180 (1974). - Type: Guinea, Lisowski 1200 (hb. Vězda, holotype!; UPS, isotype!). - Distribution: Tropical Africa.

Brasilicia ituriensis (Vězda) Farkas, comb. nova, Acta Bot. Hung. 57(1-2): 00 (2015). - Bas.: Bacidia ituriensis Vězda, Folia Geobot. Phytotax., Praha, 15: 84 (1980). - Type: Congo, Lisowski s.n. (hb. Vězda, holotype!). - Distribution: Tropical Africa (Congo).

Brasilicia olivaceorufa (Vain.) Farkas, comb. nova, Acta Bot. Hung. 57(12): 00 (2015). - Bas.: Bacidia olivaceorufa Vain., Ann. Acad. Sci. Fenn., ser. A, 15: 65 (1921). - Tax. syn.: Bacidia depokensis Zahlbr. - Type: Philippines, Robinson s.n. (TUR, holotype, not seen). - Distribution: Tropical Asia. 
Brasilicia subsimilis (Vězda) Farkas, comb. nova, Acta Bot. Hung. 57(12): 00 (2015). - Bas.: Bacidia subsimilis Vězda, Folia Geobot. Phytotax., Praha, 10: 424 (1975). - Type: Tanzania, Pócs 6379 (hb. Vězda, holotype!). - Distribution: Tropical Africa (Tanzania).

Eugeniella Lücking Sérus. et Kalb in Lücking, Flora Neotropica 103: 712 (2008). - Type species: Eugeniella psychotriae (Müll. Arg.) Lücking, Sérus. et Kalb $\equiv$ Patellaria psychotriae Müll. Arg. - Systematic position: Lecanorales, Pilocarpaceae. - Substrata: Predominantly foliicolous, (corticolous). - Number of foliicolous species: 7. - Distribution: Pantropical.

Eugeniella atrichoides (Malme) Lücking, Sérus. et Kalb, Flora Neotropica 103: 718 (2008). - Bas.: Bacidia atrichoides Malme - Type: Brazil, Malme 2287: $1 \mathrm{~b}$ (S, holotype!). - Distribution: Tropical America.

Eugeniella corallifera (Lücking) Lücking, Sérus. et Kalb, Flora Neotropica 103: 716 (2008). - Bas.: Bacidia corallifera Lücking in Lücking and Lücking. - Type: Costa Rica (Cocos Island), Lücking \& Lücking 92-1028 (CR, holotype!; Lücking, Lich. Fol. Exs. 126, isotypes!). - Exs.: Lücking, Lich. Fol. Exs. 126, 196. - Distribution: Tropical America.

Eugeniella leucocheila (Tuck.) Lücking, Sérus. et Kalb, Flora Neotropica 103: 714 (2008). - Bas.: Lecidea leucocheila Tuck., Proceedings of the American Academy of Arts and Sciences 6: 278 (1866). - Type: (corticolous). Cuba. Unknown locality, Wright s.n. (holotype, FH; isotype, BM; Wright, Lich. Cubae 208). - Tax. syn.: Pilocarpon wettsteinii Zahlbr., Denkschr. Math.-Nat. Kl. Akad. Wiss. Wien 83: 115 (1909). झ Byssoloma wettsteinii (Zahlbr.) Zahlbr., Catal. Lich. Univ. 2: 571 (1923); Lücking, Abstr. Bot. 21 (1): 96 (1997). - Type: Brazil, São Paulo, Bertioga, 5-25 m, 1901, Schiffner s.n. (holotype, W; isotype, BM). - Distribution: Tropical America.

Eugeniella micrommata (Kremp.) Lücking, Sérus. et Kalb, Flora Neotropica 103: 716 (2008). - Bas.: Lecanora micrommata Kremp. $\equiv$ Bacidia micrommata (Kremp.) R. Sant., Symb. Bot. Ups. 12(1): 459 (1952). - Type: Malaysia (Borneo), Beccari 228 (M, holotype!; G, isotype!). - Tax. syn.: Bilimbia mastophoriza Vain.; Bilimbia mastophoriza var. lacerata Vain.; Bilimbia mastophoriza var. laevior Vain. - Distribution: Tropical Africa and Asia.

Eugeniella newtoniana (Henriq.) Lücking, Sérus. et Kalb, Flora Neotropica 103: 716 (2008). - Bas.: Lecanora newtoniana Henriq., Lich. Ins. Guin.: 17 (1889). $\equiv$ Lopadium newtonianum (Henriq.) R. Sant., Symb. Bot. Ups. 12(1): 543 (1952). - Type: São Thomé \& Príncipe (São Thomé Isl.), Newton s.n. (H, holotype!; COI, isotype, not seen). - Distribution: Tropical Africa (São Thomé Isl.).

Eugeniella ortizii (Lücking) Lücking, Sérus. et Kalb, Flora Neotropica 103: 715 (2008). - Bas.: Byssoloma ortizii Lücking, Nova Hedwigia 52: 299 (1991). - Type: Costa Rica, Lücking 276 (hb. Vězda, holotype!). - Exs.: Lücking, Lich. Fol. Exs. 102. - Distribution: Tropical America. 
Eugeniella psychotriae (Müll. Arg.) Lücking, Sérus. et Kalb, Flora Neotropica 103: 714 (2008). - Bas.: Patellaria psychotriae Müll. Arg. 三 Bacidia psychotriae (Müll. Arg.) Zahlbr. - Type: Brazil, Spruce s.n. (G, holotype!). - Tax. syn.: Lecania bicolor Müll. Arg. - Exs.: Lücking, Lich. Fol. Exs. 55. - Distribution: Tropical America and Asia.

Fellhanera Vězda, Folia Geobot. Phytotax., Praha, 21: 200 (1986). - Type species: Fellhanera fuscatula (Müll. Arg.) Vězda. - Systematic position: Lecanorales, Pilocarpaceae, see Vězda, Folia Geobot. Phytotax., Praha, 21: 200 (1986). - Substrata: Ubiquitous. - Number of foliicolous species: 65. - Distribution: Cosmopolitan.

Fellhanera africana (Vězda) Lücking, The Lichenologist 33 (3): 189 (2001). - Bas.: Bacidia africana Vězda - Type: Tanzania, Pócs 6397 (hb. Vězda, holotype!). - Distribution: Tropical Africa.

Fellhanera albidocincta (Vain.) Lücking, The Lichenologist 33 (3): 191 (2001). - Bas.: Bilimbia albidocincta Vain. Annal. Acad. Sci. Fenn. 15 (6): 84 (1921). = Bacidia albidocincta (Vain.) Zahlbr., Catal. Lich. Univ. 4: 99 (1926). Type: Philippines, Robinson s.n. (TUR-Vainio 21560, holotype!). - Distribution: Tropical Asia and Australia.

Fellhanera angustispora Lücking, Trop. Bryol. 13: 143 (1997). = Fellhanera naevia (Vain.) Lücking et Cáceres.

Fellhanera atrofuscatula Herrera-Camp. et Lücking, Phyton 44 (2): 172 (2004). - Type: Herrera-Campos et al. s.n. (holotype, MEXU; isotype, F). - Distribution: Tropical America.

Fellhanera aurantiaca (Vězda) Vězda, Folia Geobot. Phytotax., Praha, 21: 214 (1986). - Bas.: Bacidia aurantiaca Vězda. - Type: Guinea, Lisowski 1248 (hb. Vězda, holotype!; UPS, isotype!). - Exs.: Kalb, Lich. Neotrop. 505. - Distribution: Tropical America and Africa.

Fellhanera avilezii Lücking in Lücking et Lücking, Herzogia 11: 154 (1995). - Type: Costa Rica (Cocos Isl.), Lücking \& Lücking 92-815 (CR, holotype!; Lücking, Lich. Fol. Exs. 135, isotypes!). - Exs.: Lücking, Lich. Fol. Exs. 135. - Distribution: Tropical America (Cocos Isl.).

Fellhanera badimioides Lücking, Lumbsch et Elix, Bot. Acta 107: 400 (1994). - Type: Costa Rica, Lücking 1301 (M, holotype!). - Distribution: Tropical America.

Fellhanera bouteillei (Desm.) Vězda, Folia Geobot. Phytotax., Praha, 21: 214 (1986). - Bas.: Parmelia bouteillei Desm. 三 Catillaria bouteillei (Desm.) Zahlbr. - Type: France, Bouteille s.n. (G, isotype, not seen). - Tax. syn.: Lecidea rubicola Crouan; Biatorina hohenbuehelii Poetsch; Patellaria leptoloma Müll. Arg.; Lecidea rubicola f. abieticola Nyl.; Trichobelonium tropicale Rehm; Mollisia copelandi Rehm; Catillaria schroederi Zahlbr.; Catillaria oospora Vain.; Catillaria oospora var. mangiferae Vain.; Catillaria epiphylla Fink. - Exs.: Anonymous, Krypt. Exs. 
Vindob. 2560; Anonymous, Reliquiae Farlow 648 (as Biatora bouteillei (Desm.) Massal.); Arnold, Lich. Monac. Exs. 403 (as Biatorina bouteillei (Desm.) Arnold); Desmazières, Plant. Crypt. N. France, 1. ed., 1595 (as Lecania bouteillei); ibid., 2. ed., 1195 (as Lecania bouteillei); Desmazières, Plant. Crypt. France 600; Kerner, Flora Exs. Austro-Hung. 2761 (as Biatorina bouteillei (Desm.) Arnold); Körber, Lich. Sel. Germ. 403 (as Biatorina bouteillei (Desm.) Arnold); Lojka, Lichenothec. Univ. 28a, 28b (as Lecidea bouteillei (Desm.) Nyl.); Lücking, Lich. Fol. Exs. 89; Rabenhorst, Lich. Europ. 856 (as Biatora bouteillei); Roumegučre, Lich. Gall. Exs. 455 (as Biatora bouteillei); Vězda, Lich. Sel. Exs. 1515 (as Catillaria bouteillei); Zahlbruckner, Lich. Rar. 128. - Distribution: Cosmopolitan.

Fellhanera bullata Kalb et Vězda in Vězda and Kalb, Nova Hedwigia 53: 219 (1991). - Type: Australia, Kalb \& Kalb (hb. Kalb, holotype!; hb. Vězda, isotype!). - Exs.: Lücking, Lich. Fol. Exs. 136. - Distribution: Tropical Asia and Australia.

Fellhanera buxi (Vězda et Vivant) Vězda, Folia Geobot. Phytotax., Praha, 21: 214 (1986). - Bas.: Bacidia buxi Vězda et Vivant. = Fellhaneropsis myrtillicola (Erichs.) Sérus. et Coppins.

Fellhanera carnea (Vězda) Vězda, Folia Geobot. Phytotax., Praha, 21: 214 (1986). - Bas.: Bacidia carnea Vězda. - Type: Guinea, Lisowski 1359 (hb. Vězda, holotype!; UPS, isotype!). - Distribution: Tropical America and Africa.

Fellhanera cateilea (Vain.) Farkas in Galloway, Tropical lichens: their systematics, conservation, and ecology: 97 (1991). इ Badimia cateilea (Vain.) Lücking, Lumbsch et Elix.

Fellhanera cenii Vězda in Schedae Lichenes Rariores Exsiccati 37 (nos 361-370): 2, no. 364 (1998). - Type: Dominica, Vězda 1996 (hb. Vězda, holotype!). - Exs.: Vězda, Lich. Rar. Exs. 364. - Distribution: Tropical America (Dominica).

Fellhanera christiansenii Sérus. et Vězda in Vězda, Nova Hedwigia 58: 130 (1994). - Type: Spain (Canary Islands), Christiansen 4885 (MSC, holotype, not seen; hb. Vězda, isotype!). - Exs.: Lücking, Lich. Fol. Exs. 137. - Distribution: Europe (Canary Islands, Italy).

Fellhanera colchica (Vězda) Llop, The Lichenologist 39 (4): 393 (2007). - Bas.: Bacidia colchica Vězda, Folia Geobot. Phytotax., Praha, 14: 203 (1979). Type: Georgia, Vězda s.n. (hb. Vězda, holotype!; Vězda, Lich. Sel. Exs. 1660, isotypes!). - Exs.: Vězda, Lich. Sel. Exs. 1660 (isotypes). - Distribution: Tethyan.

Fellhanera congesta (Vězda) Vězda, Folia Geobot. Phytotax., Praha, 21: 214 (1986). - Bas.: Catillaria congesta Vězda. - Type: Tanzania, Pócs 6397 (hb. Vězda, holotype!). - Distribution: Tropical Africa. 
Fellhanera dictyospora Lücking, Trop. Bryol. 13: 145 (1997). - Type: Costa Rica, Lücking 91-5567 (ULM, holotype!). - Distribution: Tropical America (Costa Rica).

Fellhanera dispersa Lücking, Trop. Bryol. 13: 147 (1997). - Type: Costa Rica, Lücking 92-213 (ULM, holotype!). - Distribution: Tropical America.

Fellhanera dominicana (Vain.) Vězda, Folia Geobot. Phytotax., Praha, 21: 214 (1986). - Bas.: Lecidea dominicana Vain. = Fellhanera fuscatula (Müll. Arg.) Vězda.

Fellhanera ekmanii (Vězda) Lücking in Lücking and Lücking, Herzogia 11: 155 (1995). - Bas.: Bacidia ekmanii Vězda. - Type: Cuba, Ekman 5356 (UPS, holotype!). - Distribution: Tropical America (Cuba).

Fellhanera elliottii (Vain.) Vězda, Folia Geobot. Phytotax., Praha, 21: 214 (1986). - Bas.: Lecidea elliottii Vain. = Lopadium elliottii (Vain.) Zahlbr. - Type: Lesser Antilles (St. Vincent), Elliott s.n. (TUR, holotype!). - Distribution: Tropical America and Africa.

Fellhanera emarginata Lücking, Trop. Bryol. 13: 148 (1997). - Type: Costa Rica, Lücking 91-5374 (ULM, holotype!). - Distribution: Tropical America.

Fellhanera encephalarti (Vězda) Vězda, Folia Geobot. Phytotax., Praha, 21: 214 (1986). - Bas.: Catillaria encephalarti Vězda. - Type: Congo, Lisowski s.n. (hb. Vězda, holotype!). - Distribution: Tropical Africa.

Fellhanera endopurpurea Hafellner et Vězda in Vězda and Hafellner, Nova Hedwigia 52: 76 (1991). - Type: Australia, Hafellner et al. s.n. (GZU, holotype!; Vězda, Lich. Sel. Exs. 2454, isotypes!). - Exs. Vězda, Lich. Sel. Exs. 2454. - Distribution: Tropical Australia.

Fellhanera farinosa Lücking, Nova Hedwigia, Beih. 104: 138 (1992); nom. inval., ICBN Art. 32, 36-37. = Fellhanera fuscatula (Müll. Arg.) Vězda, see Lücking, Trop. Bryol. 13: 149 (1997).

Fellhanera flavostanhopeae Lücking Flora Neotropica 103: 654 (2008). Type: Peru. Santesson \& Santesson s.n. (holotype, UPS). Distribution: Tropical America.

Fellhanera follmannii Ferraro et Vězda in Daniels et al., Flechten Follmann: 224 (1995). - Type: Argentina, Schinini 25398 (CTES, holotype!; hb. Vězda, isotype!). - Distribution: Tropical America (Argentina).

Fellhanera fragilis (Vězda) Lücking et Kalb, The Lichenologist 33 (3): 192 (2001). - Bas.: Bacidia fragilis Vězda, Folia Geobot. Phytotax., Praha, 10: 417 (1975). - Type: Tanzania, Pócs 6476 (hb. Vězda, holotype!). - Distribution: Tropical Africa.

Fellhanera fuscatula (Müll. Arg.) Vězda, Folia Geobot. Phytotax., Praha, 21: 214 (1986); Lücking, Trop. Bryol. 13: 149 (1997). - Bas.: Patellaria fuscatula

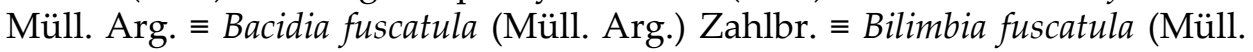
Arg.) Szatala. - Type: Venezuela, Ernst s.n. (G, lectotype!). - Tax. syn.: Patel- 
laria fuscatula var. nigricans Müll. Arg.; Patellaria rufula Müll. Arg.; Lecidea mollissiaeformis Vain.; Lecidea dominicana Vain. $\equiv$ Bacidia dominicana (Vain.) Zahlbr.; Fellhanera dominicana (Vain.) Vězda); Lecidea scottii Vain; Fellhanera farinosa Lücking, nom. inval. - Exs.: Lücking, Lich. Fol. Exs. 12 (as Fellhanera dominicana), 218; Vězda, Lich. Rar. Exs. 171. - Distribution: Pantropical.

Fellhanera ivoriensis Lücking et R. Sant., The Lichenologist 33 (2): 113 (2001). - Type: Santesson 10745 (UPS, holotypus). - Distribution: Tropical Africa.

Fellhanera lambinonii (Sérus.) Lücking et Sérus. in Sérusiaux, Lichenologist 28: 219 (1996). - Bas.: Bacidia lambinonii Sérus. = Byssoloma lambinonii (Sérus.) Sérus. - Type: Rwanda, Lambinon 75/1618 (LG, holotype!). - Tax. syn.: Bacidia michaeliana Sérus. $\equiv$ Fellhanera michaeliana (Sérus.) Sérus. - Distribution: Tropical America and Africa.

Fellhanera lisowskii (Vězda) Vězda, Folia Geobot. Phytotax., Praha, 21: 214 (1986). - Bas.: Bacidia lisowskii Vězda. - Type: Congo, Lisowski s.n. (hb. Vězda, holotype!; Vězda, Lich. Sel. Exs. 1690, isotypes!). - Exs.: Vězda, Lich. Sel. Exs. 1690 (as Bacidia lisowskii). - Distribution: Tropical America and Africa.

Fellhanera longispora Lücking, Trop. Bryol. 13: 153 (1997). - Type: Costa Rica, Lücking 91-1306 (ULM, holotype!; G, NY, UPS, VBI, isotypes!). - Distribution: Tropical America.

Fellhanera mackeei Lücking et Hürl., Bibliotheca Lichenologica 78: 255 (2001). - Type: NewCaledonia, H. S. MacKee 45609a (PC, holotype). - Distribution: Eastern Paleotropical.

Fellhanera mastothallina (Vain.) Lücking et Sérus., The Lichenologist 33 (3): 192 (2001), - Bas.: Bacidia mastothallina Vain. Ann. Acad. Sci. Fenn., ser. A, 15: 64 (1921). = Bacidina mastothallina (Vain.) Vězda in Vězda et al. Ann. Naturh. Mus. Wien 99B: 738 (1997). - Type: Philippines, Robinson \& Ramos 11900 (TUR, holotype, not seen). - Tax. syn.: Bacidia poliophaea Vain. - Distribution: Tropical Asia and Australia.

Fellhanera michaeliana (Sérus.) Sérus., Nord. J. Bot. 13: 454 (1993). - Bas.: Bacidia michaeliana Sérus. $=$ Fellhanera lambinonii (Sérus.) Lücking et Sérus.

Fellhanera microdiscus (Vain.) Vězda, Folia Geobot. Phytotax., Praha, 21: 214 (1986). - Bas.: Bilimbia microdiscus Vain. - Type: Philippines, Robinson s.n. (TUR, holotype!). - Distribution: Tropical Asia.

Fellhanera myrtillicola (Erichs.) Hafellner in Vězda, Sched. Lich. Sel. Exs., Fasc. XCV: 3, no. 2358 (1989). इ Fellhaneropsis myrtillicola (Erichs.) Sérus. et Coppins.

Fellhanera misionensis Ferraro et Lücking, Mycotaxon 73: 164 (1999); Lücking, Trop. Bryol. 13: 154 (1997). - Type: Argentina, Maruńak et al. 731 (CTES, holotype!). - Distribution: Tropical America. 
Fellhanera montana Lücking, Trop. Bryol. 13: 154 (1997). - Type: Costa Rica, Lücking 92-4762 (ULM, holotype!). - Distribution: Tropical America (Costa Rica).

Fellhanera muhlei Lücking, Trop. Bryol. 13: 155 (1997). - Type: Costa Rica, Lücking 91-4775 (ULM, holotype!). - Distribution: Tropical America (Costa Rica).

Fellhanera naevia (Vain.) Lücking et M. Cáceres, The Lichenologist 33 (3): 193 (2001). - Bas.: Bacidia naevia Vain., Ann. Acad. Sci. Fenn., ser. A, 15: 66 (1921). - Type: Philippines. Robinson 18221 (holotype, TUR; isotype, F). - Tax. syn.: Fellhanera angustispora Lücking, Trop. Bryol. 13: 143 (1997). - Distribution: Eastern paleotropics.

Fellhanera obscurata Herrera-Camp. et Lücking, Phyton 44 (2): 174 (2004). - Type: Mexico, Herrera-Campos et al. s.n. (holotype, MEXU; isotype, F). - Distribution: Tropical America.

Fellhanera paradoxa (Vězda) Vězda, Folia Geobot. Phytotax., Praha, 21: 214 (1986). - Bas.: Bacidia paradoxa Vězda. - Type: Guinea, Lisowski 1201 (hb. Vězda, holotype!; UPS, isotype!). - Distribution: Tropical America and Africa.

Fellhanera parvula (Vězda) Vězda, Folia Geobot. Phytotax., Praha, 21: 214 (1986). - Bas.: Catillaria parvula Vězda. - Type: Guinea, Lisowski 1222 (hb. Vězda, holotype!; UPS, isotype!). - Exs: Kalb, Lich. Neotrop. 10; Vězda, Lich. Sel. Exs. 2260. - Distribution: Tropical America and Africa.

Fellhanera pauciseptata (R. Sant.) Lücking, Trop. Bryol. 13: 161 (1997). - Bas.: Bacidia pauciseptata R. Sant. - Type: Colombia, von Sneidern s.n. (S, holotype!; UPS, isotype!). - Distribution: Tropical America.

Fellhanera perpusilla (Vain.) Matzer, Mycol. Pap. 171: 177 (1996). - Bas.: Lecidea perpusilla Vain. - Type: Philippines, Robinson s.n. (TUR-Vainio 23078, holotype, not seen). - Tax. syn.: Arthonia subinvisibilis Vain. - Distribution: Tropical Asia (Philippines).

Fellhanera pilomarginata Lücking, Trop. Bryol. 13: 156 (1997). - Type: Costa Rica, Lücking 92-5100 (ULM, holotype!). - Distribution: Tropical America (Costa Rica).

Fellhanera punctata Lücking, Flora Neotropica 103: 644 (2008). - Type: Brazil, Lücking 95-322 (holotype, MG). - Distribution: Tropical America.

Fellhanera rhapidophylli (Rehm) Vězda, Folia Geobot. Phytotax., Praha, 21: 214 (1986). - Bas.: Bilimbia rhapidophylli Rehm. $\equiv$ Bacidia rhapidophylli (Rehm) Zahlbr. - Type: Philippines, Raimundo, comm. Baker 1817b (S, holotype!; UPS, isotype!). - Tax. syn.: Bilimbia caryotae Vain.; ?Chlorospleniella intermixta Rehm. - Distribution: Pantropical.

Fellhanera rubida (Müll. Arg.) Lücking, Trop. Bryol. 13: 160 (1997). Bas.: Patellaria rubida Müll. Arg. - Type: Brazil, Puiggari s.n. (G, holotype!). - Distribution: Tropical America and Africa. 
Fellhanera rubrolecanorina A. B. Peña et Colín, Phyton 44 (2): 175 (2004). - Type: Herrera-Campos et al. s.n. (holotype, MEXU; isotype, F!). - Distribution: Tropical America.

Fellhanera santessonii Barillas et Lücking, Cryptogamie Bryol. Lichénol. 13: 311 (1992). - Type: Guatemala, Winkler 25 (M, holotype!). - Distribution: Tropical America.

Fellhanera semecarpi (Vain.) Vězda, Folia Geobot. Phytotax., Praha, 21: 215 (1986). - Bas.: Catillaria semecarpi Vain. - Type: Philippines, Robinson 17365 (TUR, holotype!). - Exs.: Lücking, Lich. Fol. Exs. 35, 90; Vězda, Lich. Sel. Exs. 2030 (as Catillaria semecarpi). - Distribution: Pantropical.

Fellhanera seroexspectata Sérus., Lichenologist 28: 211 (1996). - Type: Portugal (Madeira), Sérusiaux s.n. (LG, holotype!). - Distribution: Europe (Madeira).

Fellhanera sorediantha (Vězda) Vězda, Folia Geobot. Phytotax., Praha, 21: 214 (1986). - Bas.: Bacidia sorediantha Vězda. - Type: Congo, Lisowski s.n. (hb. Vězda, holotype!). - Distribution: Tropical Africa.

Fellhanera stanhopeae (Müll. Arg.) Lücking, Lumbsch et Elix, Bot. Acta 107: 400 (1994). - Bas.: Patellaria stanhopiae Müll. Arg. $\equiv$ Bacidia stanhopeae (Müll. Arg.) Zahlbr. झ Badimia stanhopeae (Müll. Arg.) Vězda. - Type: Venezuela, Ernst s.n. (G, holotype!). - Exs.: Kalb, Lich. Neotrop. 356 (as Bacidia stanhopeae); Lücking, Lich. Fol. Exs. 63; Vězda, Lich. Sel. Exs. 2307 (as Bacidia stanhopeae). - Distribution: Tropical America.

Fellhanera subfuscatula Lücking, Trop. Bryol. 13: 162 (1997). - Type: Paraguay, Balansa 4255 (G, holotype!). - Tax. syn.: ?Chlorospleniella intermixta var. gomphiae Rehm. - Exs.: Lücking, Lich. Fol. Exs. 196; Vězda, Lich. Sel. Exs. 2155, 2357 (as Fellhanera fuscatula (Müll. Arg.) Vězda). - Distribution: Pantropical.

Fellhanera sublecanorina (Nyl.) Vězda, Sched. Lich. Sel. Exs., Fasc. LXXXVII: 2, no. 2156 (1987). - Bas.: Platygrapha sublecanorina Nyl. झ Bacidia sublecanorina (Nyl.) Zahlbr. झ Bilimbia sublecanorina (Nyl.) Szatala. - Type: Angola, Welwitsch 308 (LISU, holotype, not seen; H, TUR, isotypes!). - Tax. syn.: Patellaria sprucei Müll. Arg.; Patellaria filicina Müll. Arg.; Lecidea vagula Nyl.; Lecidea atricha Vain. - Exs.: Kalb, Lich. Neotrop. 6, 357; Lücking, Lich. Fol. Exs. 36, 138; Vainio, Lich. Bras. Exs. 565 (as Lecidea atricha); Vězda, Lich. Sel. Exs. 2033, 2156, 2261. - Distribution: Pantropical.

Fellhanera submicrommata (Vězda) Lücking et Kalb, The Lichenologist 33 (3): 193 (2001). - Bas.: Bacidia submicrommata Vězda, Acta Mus. Silesiae, Opava, ser. A, 24: 124 (1975). - Type: Guinea, Lisowski 1169 (hb. Vězda, holotype!; UPS, isotype!). - Distribution: Tropical Africa.

Fellhanera substanhopeae Lücking in Ferraro and Lücking, Mycotaxon 73: 165 (1999). - Type: Brazil, Lücking 95-70 (M, holotype!). - Exs.: Lücking, Lich. Fol. Exs. 240. - Distribution: Tropical America (northern South America). 
Fellhanera subternella (Nyl.) Vězda, Folia Geobot. Phytotax., Praha, 21: 215 (1986). - Bas.: Lecidea subternella Nyl. = Bacidia subternella (Nyl.) R. Sant. - Type: São Thomé \& Príncipe (São Thomé Isl.), Newton s.n. (H, holotype!; COI, isotype, not seen). - Tax. syn.: Patellaria fulvula Müll. Arg.; ?Patellaria pulverulenta Müll. Arg.; ?Patellaria aeruginosula Müll. Arg.; ?Lecanora sprucei Bab. ex Nyl. - Distribution: Pantropical.

Fellhanera subtilis (Vězda) Diederich et Sérus. in Sérusiaux, Mem. Soc. Roy. Bot. Belg. 12: 142 (1990). - Bas.: Bacidia subtilis Vězda, Preslia, Praha, 33: 367 (1961). $\equiv$ Arthonia subtilis (Vězda) Vězda. - Type: Czech Republic, Vězda (PRM, holotype; BM, DUKE, GZU, M UPS, isotypes). - Exs.: Vězda, Lich. Sel. Exs. 111 (as Arthonia subtilis). - Distribution: Europe.

Fellhanera tasmanica Lücking et Elix, The Lichenologist 33 (3): 204 (2001). - Type: Australia, Elix 5688 (CANB, holotypus; hb. Lücking, isotypus). - Distribution: Australia.

Fellhanera tubulifera Rain. Schub. et Lücking, Willdenowia 33 (2): 461 (2003). - Type: Costa Rica, Schubert 234 (CR; isotypes: ULM, hb. Schubert). Distribution: Tropical America (Costa Rica).

Fellhanera tricharioides Lücking et R. Sant. in Lücking and Kalb ined., Bot. Jahrb. Syst. 122: 11 (2000); nom. inval., ICBN Art. 32, 36-37.

Fellhanera tricharioides Lücking et R. Sant., Flora Neotropica 103: 661 (2008). - Type: Trinidad and Tobago, Trinidad, Thaxter 41 (UPS, holotype). Distribution: Tropical America.

Fellhanera vandenberghenii (Sérus.) Vězda, Folia Geobot. Phytotax., Praha, 21: 215 (1986). - Bas.: Catillaria vandenberghenii Sérus. - Type: Zimbabwe, Vanden Berghen s.n. (LG, holotype!; hb. Vězda, isotype!). - Distribution: Tropical Africa.

Fellhanera verrucifera Lücking, Trop. Bryol. 13: 163 (1997). - Type: Costa Rica, Lücking 92-60 (ULM, holotype!). - Exs.: Lücking, Lich. Fol. Exs. 219. Distribution: Tropical America.

Fellhanera verrucifera var. nigrescens Lücking, Flora Neotropica 103: 644 (2008). - Type: Guyana, Lücking 96-3148 (holotype, US; isotype, hb. Lücking). - Distribution: Tropical America.

Fellhanera viridis Lücking, Nova Hedwigia, Beih. 104: 136 (1992); Trop. Bryol. 13: 163 (1997). - Type: Costa Rica, Lücking 87-272 (ULM, holotype!). Distribution: Tropical America (Costa Rica).

Fellhanera winkleriana Lücking, Nova Hedwigia 52: 298 (1991). - Type: Costa Rica, Lücking 696 (LG, holotype!). - Distribution: Tropical America (Costa Rica).

Fellhanera wirthii (Vězda) Vězda, Folia Geobot. Phytotax., Praha, 21: 215 (1986). - Bas.: Bacidia wirthii Vězda. - Type: Congo, Lisowski s.n. (hb. Vězda, holotype!). - Distribution: Tropical Africa. 
Fellhaneropsis Sérus. et Coppins in Sérusiaux, Lichenologist 28: 198 (1996). - Type species: F. myrtillicola (Erichs.) Sérus. et Coppins (holotype). Systematic position: Lecanorales, Pilocarpaceae, see Sérusiaux, Lichenologist 28: 199 (1996). - Substrata: Foliicolous and corticolous. - Number of foliicolous species: 4. - Distribution: Europe and northern Asia and Australia.

Fellhaneropsis australiana Lücking, The Lichenologist 33 (3): 205 (2001). - Type: Australia, Elix 5688 (CANB, holotype; hb. Lücking, isotype). - Distribution: Australia.

Fellhaneropsis kurokawana Thor, Lücking et Matsumoto, Symb. Bot. Ups. 32 (3): 41 (2000). - Type: Japan, Matsumoto 2058 (HIRO, holotype!; TNS, isotype!). - Distribution: Tropical Asia (Japan).

Fellhaneropsis myrtillicola (Erichs.) Sérus. et Coppins in Sérusiaux, $\mathrm{Li}-$ chenologist 28: 199 (1996). - Bas.: Bacidia myrtillicola Erichs. इ Fellhanera myrtillicola (Erichs.) Hafellner. - Type: Germany, Erichsen s.n. (HBG, holotype!). - Tax. syn.: Bacidia myriocarpa Erichs.; Bacidia buxi Vězda et Vivant; Bacidia gorgonea Vězda et Poelt. - Exs.: Vězda, Lich. Sel. Exs. 1161, 1516 (as Bacidia buxi). - Distribution: Europe.

Fellhaneropsis rhododendri Aptroot, Lichenologist 44 (4): 442 (2012). Type: The Netherlands, A. Aptroot 69866 (BR, holotypus; ABL, isotypus). Distribution: Europe.

Scoliciosporum Massal., Richerche sull' Autonomia dei Licheni Crostosi: 104 (1852). - Type species: S. holomelaenum (Flk.) Massal. (lectotype). - Systematic position: Lecanorales, Scoliciosporaceae. - Substrata: Ubiquitous. Number of foliicolous species: 2. - Distribution: Cosmopolitan.

Scoliciosporum curvatum Sérus., Nord. J. Bot. 13: 458 (1993). - Type: France, Sérusiaux s.n. (LG, holotype!; Lücking, Lich. Fol. Exs. 44, isotypes!). Exs.: Lücking, Lich. Fol. Exs. 44. - Distribution: Europe.

Scoliciosporum lividum Malcolm et Vězda, Folia Geobot. Phytotax., Praha, 31: 266 (1996). - Type: New Zealand, Malcolm 1166 (CHR 486639, holotype, not seen; hb. Vězda, isotype!). - Distribution: Tropical Australia (New Zealand).

Szczawinskia Funk, Syesis 16: 85 (1983); Aptroot et al., Biblioth. Lichenol. 64: 191. (1997); Holien and Tønsberg, Lichenologist 34: 369. (2002). - Type species: Szczawinskia tsugae Funk (holotype). - Systematic position: Lecanorales, Pilocarpaceae. - Substrata: Corticolous and foliicolous. - Number of foliicolous species: 3. - Distribution: Disjunct cosmopolitan.

Szczawinskia foliicola Holien et Tønsberg, The Lichenologist 34(5): 372 (2002); Aptroot et al., Biblioth. Lichenol. 64: 191 (1997). - Type: Papua New Guinea, Sérusiaux 14023 (LG, holotype, B, isotype). - Distribution: Eastern paleotropics (Papua New Guinea). 
Szczawinskia permira (Vězda) Farkas, comb. nova, Acta Bot. Hung. 57(12): 00 (2015). - Bas.: Bacidia permira Vězda, Folia Geobot. Phytotax., Praha, 10: 419 (1975). = Uluguria permira (Vězda) Vězda, Czech Mycology 56 (1-2): 150 (2004). - Type: Tanzania, Pócs 6550 (hb. Vězda, holotype!). - Distribution: Tropical Africa (Tanzania).

Szczawinskia tsugae Funk, Syesis 16: 86 (1983); Flora Neotropica 103: 617 (2008). - Type: U.S.A. (Alaska), Curtis s.n. (DAVFP 21966, holotype!). Tax. syn.: Micarea clavopycnidiata Brodo et Tønsberg. - Distribution: Disjunct cosmopolitan (North and Central America).

Uluguria Vězda, Czech Mycol. 56(1-2): 150 (2004). - Type species: Uluguria permira (Vězda) Vězda. $\equiv$ Bacidia permira Vězda. $=$ Szczawinskia Funk.

Uluguria permira (Vězda) Vězda, Czech Mycol. 56(1-2): 150 (2004). इSzczawinskia permira (Vězda) Farkas, comb. nova.

Woessia D. Hawksw. et Poelt, Pl. Syst. Evol. 154: 207 (1986). - Excluded, no foliicololus species known.

Woessia apiahica (Müll. Arg.) Sérus., Lichenologist 28: 224 (1996). $\equiv \mathbf{B a}$ cidina apiahica (Müll. Arg.) Vězda.

Woessia arvidssonii Sérus., Biblioth. Lichenol. 58: 416 (1995). „Bacidina arvidssonii (Sérus.) Lücking.

Woessia canariensis (Lumbsch et Vězda) Sérus., Lichenologist 28: 224 (1996). इBacidina canariensis Lumbsch et Vězda.

Woessia defecta (Vězda) Sérus., Biblioth. Lichenol. 58: 420 (1995). $\equiv B \boldsymbol{B}$ cidina defecta Vězda.

Woessia pseudohyphophorifera Lücking et Sérus. in Sérusiaux, Biblioth. Lichenol. 58: 422 (1995). Bacidina pseudohyphophorifera (Lücking et Sérus.) Lücking.

Woessia vasakii (Vězda) Sérus., Lichenologist 28: 224 (1996). $\equiv$ Bacidina vasakii (Vězda) Vězda.

Acknowledgements - The work was supported by the Hungarian Scientific Research Fund (OKTA K 81232).

\section{REFERENCES}

Ekman, S. (1996): The corticolous and lignicolous species of Bacidia and Bacidina in North America. - Opera Bot. 127: 1-148.

Ekman, S. (2001): Molecular phylogeny of the Bacidiaceae (Lecanorales, lichenized Ascomycota). - Mycol. Res. 105(7): 783-797.

Lumbsch, H. T. and Huhndorf, S. M. (2010): Myconet 14: Outline of Ascomycota 2009. Notes on Ascomycete systematics. Nos. 4751-5113. - Fieldiana, Life Earth Sci. 1: 1-64. 
Lücking, R. (1998): Foliicolous lichens and their lichenicolous fungi collected during the "Smithsonian International Cryptogamic Expedition 1996" to Guyana. - Trop. Bryol. 15: $45-76$

Lücking, R. (2008): Foliicolus lichenized fungi. - Flora Neotrop. Monogr. 103: 1-867.

Lücking, R. and Martínez Colín, P. (2004): Rapid lichen guides: foliicolous lichens of the world. - The Field Museum, Chicago, mscr.

Lücking, R., Cáceres, M. E. S., Kalb, K. and Sérusiaux, E. (2001): Studies in Bacidia sensu lato (lichenized Ascomycetes: Lecanorales). II. Six new combinations in Fellhanera Vězda. - Lichenologist 33(3): 189-194.

Lücking, R., Farkas, E., Sérusiaux, E. and Sipman, H. J. M. (2000): Checklist of foliicolous lichens and their lichenicolous fungi. Part I. Foliicolous lichens. - http://www.bio.uni-bayreuth.de/planta2/ass/robert/lichens/checkfol.html.

Santesson, R. (1952): Foliicolous lichens I. A revision of the taxonomy of the obligately foliicolous, lichenized fungi. - Symb. Bot. Upsal. 12(1): 1-590.

Sérusiaux, E. (1993): New taxa of foliicolous lichens from Western Europe and Macaronesia. - Nordic J. Bot. 13: 447-467.

Thiers, B. (2013): Index Herbariorum: a global directory of public herbaria and associated staff. New York Botanical Garden's Virtual Herbarium. - http://sweetgum.nybg.org/ih/.

Vězda, A. (1974): Foliicole Flechten aus der Republik Guinea (W-Africa). II. - Acta Mus. Silesiae, ser. A, 23: 173-190.

Vězda, A. (1975a): Foliicole Flechten aus der Republik Guinea (W-Afrika). III. - Acta Mus. Silesiae, ser. A, 24: 117-126.

Vězda, A. (1975b): Foliicole Flechten aus Tanzania (Ost-Afrika). - Folia Geobot. Phytotax., Praha, 10: 383-432.

Vězda, A. (1980): Foliicole Flechten aus Zaire. Die Arten der Sammelgattungen Catillaria und Bacidia. - Folia Geobot. Phytotax., Praha, 15: 75-94.

Vězda, A. (1986): Neue Gattungen der Familie Lecideaceae s. lat. (Lichenes). - Folia Geobot. Phytotax., Praha, 21: 199-219. 